\title{
A Stochastic Geometry Approach to Modeling Time Hopping Based TDOA in 3D Indoor localization
}

\author{
Mingyuan Fan \\ Beijing University of Posts and \\ Telecommunications \\ Beijing 100876, China \\ fanmingyuan@bupt.edu.cn \\ Buyun Jia \\ Beijing University of Posts and \\ Telecommunications \\ Beijing 100876, China
}

\author{
Fuxing Yang \\ Beijing University of Posts and \\ Telecommunications \\ Beijing 100876, China
}

\author{
Bingxue Leng \\ Beijing University of Posts and \\ Telecommunications \\ Beijing 100876, China
}

\author{
Zhongliang Deng \\ Beijing University of Posts and \\ Telecommunications \\ Beijing 100876, China
}

\begin{abstract}
Indoor localization has greatly leveraged applications regarding to location based service (LBS), which witnesses ever-increasing impact on human life. Time difference of arrival (TDOA) is one of the most widely used technique for indoor localization because of its low complexity and high positioning accuracy. However, the strong multiple access interference (MAI) is a crucial issue that affects the accuracy of TDOA in indoor positioning, in order to mitigate interference, we incorporate time-hopping (TH) in TDOA technology, which can effectively reduce the interference at the transmitting side. Particularly, we propose an analytical model to evaluate the performance of the TH based TDOA in 3D indoor scenario by using the tool of stochastic geometry. 'rough the proposed model, we obtain some easy-to-use expressions for key performance metrics. The analytical results include the average interference, the success probability (i.e. the probability of a base station (BS) that can successfully participate in the localization procedure), and the number of effective serving BSs that has successfully participated in. In addition, some special cases are analyzed in detail, which reveals interesting insights. All the analytical results in this paper are validated through Monte Carlo simulations.
\end{abstract}

\section{CCS Concepts}

-Networks $\rightarrow$ Network performance modeling; Network performance analysis; Network simulations; Physical topologies;

\section{Keywords}

indoor localization; time hopping; time difference of arrival; 3D stochastic geometry

Permission to make digital or hard copies of all or part of this work for personal or classroom use is granted without fee provided that copies are not made or distributed for profit or commercial advantage and that copies bear this notice and the full citation on the first page. Copyrights for components of this work owned by others than ACM must be honored. Abstracting with credit is permitted. To copy otherwise, or republish, to post on servers or to redistribute to lists, requires prior specific permission and/or a fee. Request permissions from Permissions@acm.org.

ICTCE 2019, November 9-12, 2019, Tokyo, Japan @ 2019 Association for Computing Machinery. ACM ISBN 978-1-4503-7180$3 / 19 / 11 \ldots \$ 15.00$

https://doi.org/10.1145/3369555.3369575

\section{INTRODUCTION}

Indoor localization is an important component of many applications in smart city like navigation in hospital, shopping mall navigation, fire emergency rescue, etc. It locates objects or people inside a building using radio waves, magnetic fields, acoustic signals, or other sensory information collected by mobile devices [1]. Due to building occlusion and other reasons, it is difficult for global positioning system (GPS) to achieve highprecision indoor positioning. Therefore, the solution with the characteristics of high reliability, high precision, easy installation and cheap integrated for indoor localization is imperatively needed.

A lot of techniques for indoor localization are proposed, such as methods based on received signal strength (RSS), time-of-arrival (TOA), time-difference-of-arrival (TDOA), or angle-of-arrival (AOA). Among all these techniques, TDOA has been widely used since it is easy to install and cheap to integrate. However, TDOA based indoor location is seriously disturbed by multiple access interference (MAI) [2]. To overcome the MAI problem, lots of researches have been done. In [3], the authors analyze the performance of general self correlation peak detection, and introduce a new method of anti-MAI self correlation peak detection, which can improve $15 \mathrm{~dB}$ of the receiver's capability of anti MAI. Authors in [4] introduce a new initial acquisition and cell search algorithm with implicit MAI cancelation. However, all these researches focus on anti-MAI at the receiver (the object to be positioned), while it is also necessary to make improvements from the transmitter to reduce the effects of MAI. Therefore, timehopping $(\mathrm{TH})$ is introduced as an effective and easy implemented way to reduce MAI at the transmitter in this paper.

Stochastic geometry has been widely used as an analytical approach to model and quantify the key metrics in cellular networks for its mathematical tractability and modeling accuracy. In stochastic geometry analysis, positions of base stations (BSs) are modeled from certain stochastic point process [5]. Authors of [6] have proved the accuracy of stochastic geometry model, and they point out that the stochastic geometry model may better capture the increasingly opportunistic and dense placement of base stations in future networks. With the rapid development of economy and society, the evolution trend of future network is extending to indoor three dimensional (3D) space. In [7], a new 3D model for cellular network which considers the attenuation effect of floors and walls is proposed. The stochastic geometry is introduced to analyze the localization fundamentals in [8] and it 
makes evident that localization performance is limited by the interference from the BSs participating in the localization procedure. However, the research in [8] is in 2D space. In our work, we give a specific analysis of TDOA technology for indoor localization in 3D space.

In this paper, we conduct a stochastic geometry analysis to model and evaluate the performance of indoor localization with $\mathrm{TH}$ based TDOA technology in 3D space. Firstly, we derive the expression of average interference to prove the proposed $\mathrm{TH}$ based TDOA is effective for reducing the interference. Then we define two performance metrics: the success probability and the average number of effective serving BSs, and the closed-form expressions of the two metrics are derived. Particularly, some special cases are researched which illustrate interesting insights. This paper is organized as follows. Section II describes the system model. The localization performances which include the average interference, success probability and the average effective serving BS number are analyzed in Section III. Simulation results are presented in Section IV. Finally, Section V concludes this paper.

\section{SYSTEM MODEL}

\subsection{Network Model}

The cellular network model consists of BSs are arranged according to a homogeneous Poisson point process (PPP) $\Phi$ of intensity $\lambda$ in 3D Euclidean space $\mathrm{R}^{3}$. Fig. 1 has shown a sketch of the distribution of BSs.

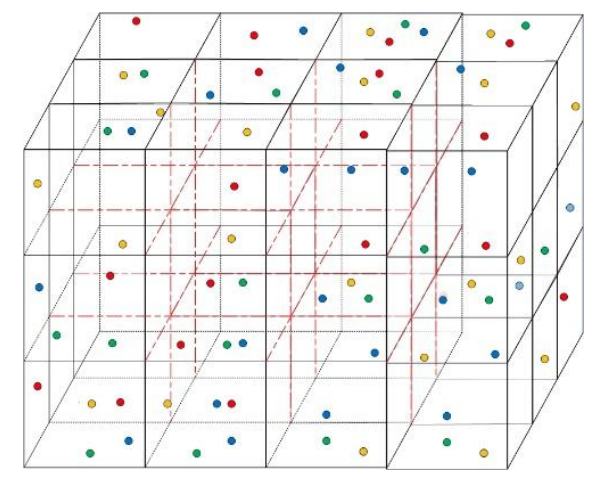

Figure 1. A topology of 3D Poisson distributed base stations.

The standard power loss propagation model is used with path loss exponent $\alpha$ and $\alpha>3$ [9]. Considering an indoor environment in $3 \mathrm{D}$, we use the path loss model refined in [10], which takes into account the penetration loss of floors and walls. The signal frequency is assumed to be $2.4 \mathrm{GHz}$. In this case, the path loss is expressed in $\mathrm{dB}$ as follows

$$
\operatorname{PL}(r)=\operatorname{PL}\left(r_{0}\right)+10 \alpha \log _{10}\left(\frac{r}{r_{0}}\right)+A F(r),
$$

where $r$ is the distance between the tagged receiver and tagged BS. $P L\left(\mathrm{r}_{0}\right)$ is the path loss of the first meter $\left(r_{0}=1 \mathrm{~m}\right), P L\left(\mathrm{r}_{0}\right)=40 \mathrm{~dB}$ at $2.4 \mathrm{GHz} . A F(\mathrm{r})$ is the average walls and floors attenuation factor [11], which is given in $\mathrm{dB}$ as

$$
\mathrm{AF}(\mathrm{r})=10 \log _{10} \text { (ur), }
$$

where $u$ is a constant attenuation per unit of path length. Based on equation (1-2), the value of the signal attenuation due to the path loss can be presented as $\mathrm{Kr}^{-(\alpha+1)}$, where $K$ is a coefficient given by $K=r_{0}^{\alpha} /\left(u 10^{P L\left(r_{0}\right) / 10}\right)$.

As far as random channel effects such as fading and shadowing, we assume that the tagged BS and tagged receiver experience only Rayleigh fading $h$ and $h \sim \operatorname{exp(1).~In~this~case,~}$ the received power at the typical receiver at distance $r$ from the tagged BS is

$$
\mathrm{P}_{\mathrm{r}}=\mathrm{P}_{\mathrm{t}} \cdot \mathrm{h} \cdot \mathrm{K} \cdot \mathrm{r}^{-(\alpha+1)} \text {. }
$$

\subsection{Time Hopping based TDOA}

In the TDOA system, the transmitted pulses from spatially separated BSs arrive at the object to be positioned $o_{p}$ at different times, and the absolute transmission time of the signal from each BS to $o_{p}$ is measured to calculate the distance difference between each pair of BSs. The distance difference further convert into a hyperboloid. In 3D positioning, at least three pairs of BSs (four different BSs) are required. The position of $o_{p}$ is the intersection of the corresponding hyperboloids.

By using time hopping, the channel interval is divided into $n_{s f}$ subframes $\left\{\mathrm{SF}_{1}, \mathrm{SF}_{2}, \cdots \mathrm{SF}_{\mathrm{n}_{\mathrm{sf}}}\right\}$, and the $\mathrm{BSs}$ are also divided into $n_{s f}$ collections $\left\{\mathrm{C}_{1}, \mathrm{C}_{2}, \cdots \mathrm{c}_{\mathrm{n}_{\mathrm{sf}}}\right\}$. All the BSs in collection $C_{i}$ transmit in the corresponding subframe $\mathrm{SF}_{\mathrm{i}}$. The mechanism of the time hopping based TDOA in this paper can be depicted as follows

- A random mark, which is uniformly distributed between 1 and $n_{s f}$, is given to each $\mathrm{BS}$ at the beginning of every $\mathrm{TH}$ period. The BSs with mark $i$ belong to $C_{i}$ and will transmit on subframe $S F_{i} . C_{i}$ follows PPP distribution of intensity $\lambda_{\mathrm{i}}=\frac{\lambda}{\mathrm{n}_{\mathrm{sf}}}$ [5]. As shown in Fig. 2 and Fig. 1, the different colors represent different subframes and the BSs in the corresponding collections. There is no interference between BSs in different collections since their corresponding subframes are orthogonal.

- According to equation (3), the closer the BS to the receiver, the stronger the signal is, which means the location information will be more accuracy. In this case, we choose the BSs in each collection which are closest to the positioned object as the serving BSs. Then, in the 3D scenario, $n_{s f}$ can not be less than 4 .

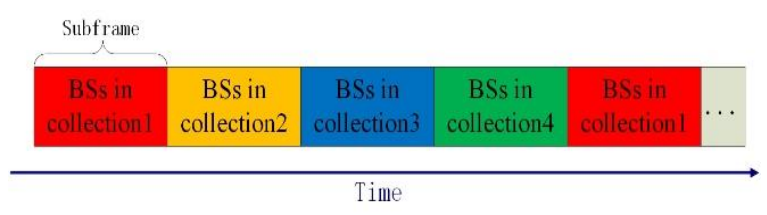

Figure 2. Sketch of subframe allocation, $n_{s f}=4$.

\section{LOCALIZATION PERFORMANCE}

Localization performance fundamentally depends on the number of serving BSs and their accuracies. These two factors are highly interdependent. Both the number of effective serving BSs whose signals arrive with sufficiently - high SINRs to participate in the localization procedure and the quality of their positioning observations are strongly correlated to the interference. Therefore, in this section, firstly, we will analyze the average interference, then the quality of the serving BSs' positioning observations is 
evaluated by defining a metric, success probability. The success probability is defined as the probability that the signal from a serving BS is successfully detected. It can also be considered as the probability of a serving BS can successfully participate in the localization procedure. Finally, we calculate the number of effective serving BSs whose signals arrive with sufficiently - high SINRs. Since the positions of BSs in different collections are independent identically distributed, therefore, a typical collection denoted as $C_{0}$ is analyzed for representation. Assume there is a object $o_{p}$ to be positioned, without loss of generation, we assume $o_{p}$ is the origin.

\subsection{Interference Analysis}

According to the system model, the interference received at $o_{p}$ from the BSs in collection $C_{0}$ can be expressed as

$$
\mathrm{I}_{\mathrm{r}}=\sum_{\mathrm{BS}_{\mathrm{i}} \in \mathrm{C}_{0} \backslash \backslash \mathrm{BS}_{1}} \mathrm{P}_{\mathrm{t}} \mathrm{h}_{\mathrm{i}} \mathrm{Kr}_{\mathrm{i}}^{-(\alpha+1)} \text {, }
$$

where $\mathrm{BS}_{1}$ is the closest $\mathrm{BS}$ to the object, which also means $\mathrm{BS}_{1}$ is the serving BS of collection $C_{0}$, and the distance between $\mathrm{BS}_{1}$ and $o_{p}$ is denoted as $r_{l} . h_{i}$ and $r_{i}$ are respectively the fading and distance between other BSs in $C_{0}$ (interference BSs) and $o_{p}$.

The average interference received at $o_{p}$ can be calculated as [12]

$$
\mathrm{E}\left[\mathrm{I}_{\mathrm{r}}\right]=\mathrm{E}_{\mathrm{r}}\left[-\left|\frac{\mathrm{d}}{\mathrm{ds}} \mathcal{L}_{J_{r}}(\mathrm{~s})\right|_{\mathrm{s}=0}\right],
$$

where $\mathcal{L}_{\mathcal{J}_{r}}(\mathrm{~s})$ is the Laplace transform of interference, and it can be calculated as follows

$$
\begin{aligned}
& \mathcal{L}_{J_{r}}(\mathrm{~s})=\mathrm{E}\left[\mathrm{e}^{-\mathrm{sIr}}\right] \\
& \stackrel{(a)}{=} \mathrm{E}_{\mathrm{C}_{\circledast}, \mathrm{h}_{\mathrm{i}}}\left[\mathrm{e}^{-\mathrm{s} \sum_{\mathrm{BS}_{\mathrm{i}} \in \mathrm{C}_{0} / \mathrm{BS}_{1}} \mathrm{P}_{\mathrm{t}} \mathrm{Kh}_{\mathrm{i}} \mathrm{r}_{\mathrm{i}}^{-(\alpha+1)}}\right] \\
& =\mathrm{E}_{\mathrm{C}_{\circledast}, \mathrm{h}_{\mathrm{i}}}\left[\prod_{\mathrm{BS}_{\mathrm{i}} \in \mathrm{C}_{0} / \mathrm{BS}_{1}} \mathrm{e}^{-\mathrm{SP}_{\mathrm{t}} \mathrm{Kh}_{\mathrm{i}} \mathrm{r}_{\mathrm{i}}^{-(\alpha+1)}}\right] \\
& =\mathrm{E}_{\mathrm{C}_{\mathbb{}}}\left[\prod_{\mathrm{BS}_{\mathrm{i}} \in \mathrm{C}_{0} / \mathrm{BS}_{1}} \mathrm{E}_{\mathrm{h}_{\mathrm{i}}}\left[\mathrm{e}^{-\mathrm{sP}_{\mathrm{t}} \mathrm{Kh}_{\mathrm{i}} \mathrm{r}_{\mathrm{i}}^{-(\alpha+1)}}\right]\right] \\
& \stackrel{(b)}{=} \mathrm{E}_{\mathrm{C}_{\varpi}}\left[\prod_{\mathrm{BS}_{\mathrm{i}} \in \mathrm{C}_{0} / \mathrm{BS}_{1}} \frac{1}{\mathrm{sP}_{\mathrm{t}} \mathrm{Kr}_{\mathrm{i}}^{-(\alpha+1)}+1}\right] \\
& =\mathrm{e}^{-4 \pi \lambda_{0} \int_{\mathrm{r}_{1}}^{\infty} \frac{\mathrm{sP}_{\mathrm{t}} \mathrm{K}}{\mathrm{sP}_{\mathrm{t}} \mathrm{K}+\mathrm{r}_{\mathrm{i}}^{(\alpha+1)}} \mathrm{r}_{\mathrm{i}}^{2} \mathrm{dr} \mathrm{r}_{\mathrm{i}}} \text {, }
\end{aligned}
$$

where step (a) comes after plugging in the expression of $I_{r}$ in equation (4). Step (b) follows the i.i.d. distribution of $h_{i}$. The last step follows from the probability generating functional (PGFL) [5] of the PPP, which states for some function $f(x)$ that $\mathrm{E}\left[\prod_{\mathrm{x} \in \Phi} \mathrm{f}(\mathrm{x})\right]=\exp \left(-\lambda \int_{\mathrm{R}}(1-\mathrm{f}(\mathrm{x})) \mathrm{dx}\right)$. The integration limits are from $r_{1}$ to $\infty$ since the closest interferer is at least at a distance $r_{1}$ from $o_{p} \cdot \lambda_{0}$ in the last step represents the density of $C_{0}$, and according to section $\mathrm{I}, \lambda_{0}=\frac{\lambda}{n_{s f}}$.

Therefore, the average interference can be derived as

$$
\begin{aligned}
\mathrm{E}\left[\mathrm{I}_{\mathrm{r}}\right] & =\mathrm{E}_{\mathrm{r}_{1}}\left[-\left|\frac{\mathrm{d}}{\mathrm{ds}} \mathcal{L}_{J_{r}}(\mathrm{~s})\right|_{\mathrm{s}=0}\right] \\
& =\mathrm{E}_{\mathrm{r}_{1}}\left[\frac{4 \pi \lambda_{0} \mathrm{P}_{\mathrm{t}} \mathrm{Kr}_{1}^{-(\alpha-2)}}{\alpha-2}\right] \\
& =\int_{0}^{\infty} \frac{4 \pi \lambda_{0} \mathrm{P}_{\mathrm{t}} \mathrm{Kr}_{1}^{-(\alpha-2)}}{\alpha-2} \mathrm{f}\left(\mathrm{r}_{1}\right) \mathrm{dr}_{1}
\end{aligned}
$$

$$
\begin{aligned}
& \stackrel{(a)}{=} \int_{0}^{\infty} 4 \pi \mathrm{r}_{1}^{2} \lambda_{0} \frac{4 \pi \lambda_{0} P_{t} K r_{1}^{-(\alpha-2)}}{\alpha-2} \mathrm{e}^{-\frac{4}{3} \pi \mathrm{r}_{1}^{3} \lambda_{0}} \mathrm{dr}_{1} \\
& \stackrel{(b)}{=} \int_{0}^{\infty} \frac{16 \pi^{2} \frac{\lambda^{2}}{\mathrm{n}_{\mathrm{sf}}^{2}} \mathrm{P}_{\mathrm{t}} \mathrm{Kr}_{1}^{-(\alpha-4)}}{\alpha-2} \cdot \mathrm{e}^{-\frac{4}{3} \pi \mathrm{r}_{1}^{3} \frac{\lambda}{\mathrm{n}_{\mathrm{sf}}}} \mathrm{dr}_{1},
\end{aligned}
$$

where step (a) is after expressing the probability density function (PDF) of $r_{1}$, and step (b) comes from $\lambda_{0}=\frac{\lambda}{n_{s f}}$. According to [5], the PDF of $r_{1}$ is expressed as

$$
\mathrm{f}_{\mathrm{r}}\left(\mathrm{r}_{1}\right)=4 \pi \mathrm{r}_{1}^{2} \lambda_{0} \cdot \mathrm{e}^{-\frac{4}{3} \pi \mathrm{r}_{1}^{3} \lambda_{0}}
$$

It can be proved that the average interference is a decreasing function with respect to the subframe number $n_{s f}$ by calculating the partial derivative of the average interference with respect to $n_{s f}$, and $\frac{\partial \mathrm{E}\left[\mathrm{I}_{\mathrm{r}}\right]}{\partial \mathrm{n}_{\mathrm{sf}}}$ is derived as follows

$$
\begin{aligned}
\frac{\partial \mathrm{E}\left[\mathrm{I}_{\mathrm{r}}\right]}{\partial \mathrm{n}_{\mathrm{sf}}} & =\int_{0}^{\infty} \frac{16 \pi^{2} \frac{\lambda^{2}}{\mathrm{n}_{\mathrm{sf}}^{3}} \mathrm{P}_{\mathrm{t}} \mathrm{Kr}_{1}^{-(\alpha-4)}}{\alpha-2} \cdot \mathrm{e}^{-\frac{4}{3} \pi \mathrm{r}_{1}^{3} \frac{\lambda}{\mathrm{n}_{\mathrm{sf}}}} \\
& \cdot\left(\frac{\frac{4}{3} \pi r_{1}^{3} \lambda}{n_{s f}}-2\right) d r_{1} .
\end{aligned}
$$

Since $\frac{\frac{4}{3} \pi r_{1}^{3} \lambda}{n_{s f}}$ is the average number of BSs in volume of $\frac{4}{3} \pi r_{1}^{3}$ in $3 D$ space [2], and $r_{1}$ is the closest $\mathrm{BS}$, therefore $\frac{\frac{4}{3} \pi \mathrm{r}_{1}^{3} \lambda}{\mathrm{n}_{\mathrm{sf}}}=1$, then, $\frac{\partial \mathrm{E}\left[\mathrm{I}_{\mathrm{r}}\right]}{\partial \mathrm{n}_{\mathrm{sf}}}<0$, which means the average interference is a decreasing function with respect to the subframe number $n_{s f}$.

\subsection{Success Probability and Effective Serving BS Number Evaluation}

Based on the definition of success probability, it is modeled as the probability of SINR higher than a pre-defined threshold $\gamma$, which is expressed as follows

$$
\mathrm{p}_{\mathrm{s}}=\mathrm{P}[\operatorname{SINR}>\gamma] .
$$

The SINR received at $o_{p}$ is expressed as

$$
\operatorname{SINR}=\frac{\mathrm{P}_{\mathrm{t}} \mathrm{h}_{1} \mathrm{Kr}_{1}^{-(\alpha+1)}}{\mathrm{I}_{\mathrm{r}}+\sigma^{2}},
$$

where $h_{1}$ represents the channel fading between the serving BS $B S_{l}$ and the object $o_{p}$, and $r_{1}$ is the distance between them. $I_{r}$ is the interference in equation (4), and $\sigma^{2}$ is the noise power.

By plugging equation (11) to equation (10), the success probability is calculated as

$$
\begin{aligned}
p_{s} & =P\left[\operatorname{SINR}>\gamma \mid r_{1}\right] \\
& =E_{h_{\mathbb{1}}, I_{r}}\left[\frac{P_{t} K h_{1} r_{1}^{-(\alpha+1)}}{I_{r}+\sigma^{2}}>\gamma \mid r_{1}\right] \\
& =E_{I r}\left[E_{h_{1}}\left[h_{1}>\frac{\gamma\left(I_{r}+\sigma^{2}\right) r_{1}^{\alpha+1}}{P_{t} K}\right] \mid r_{1}\right] \\
& \stackrel{(a)}{=} E_{I_{r}}\left[e^{\left.-\frac{\gamma\left(I_{r}+\sigma^{2}\right) r_{1}^{\alpha+1}}{P_{t} K} \mid r_{1}\right]}\right. \\
& =\int_{0}^{\infty} e^{-\frac{\gamma \sigma^{2} r_{1}^{\alpha+1}}{P_{t} K} \mathcal{L}_{\jmath_{r}}\left(\frac{\gamma r_{1}^{\alpha+1}}{P_{t} K}\right) f\left(r_{1}\right) d r_{1}} \\
& \stackrel{(b)}{=} \int_{0}^{\infty} 4 \pi r_{1}^{2} \lambda_{0} \cdot e^{-\frac{\gamma \sigma^{2} r_{1}^{\alpha+1}}{P_{t} K} \mathcal{L}_{J_{r}}}\left(\frac{\gamma r_{1}^{\alpha+1}}{P_{t} K}\right) \cdot e^{-\frac{4}{3} \pi r_{1}^{3} \lambda_{0}} d r_{1},
\end{aligned}
$$

where step (a) follows from $h_{1} \sim \exp (1)$, and step (b) comes after expressing the PDF of $r_{1}$ which has been given in equation (8). 
$\mathcal{L}_{J_{r}}\left(\frac{\gamma \mathrm{r}_{1}^{\alpha+1}}{\mathrm{P}_{\mathrm{t}} \mathrm{K}}\right)$ is the Laplace transform of the interference, and it can be obtained by plugging $\mathrm{s}=\frac{\gamma \mathrm{r}_{1}^{\alpha+1}}{\mathrm{P}_{\mathrm{t}} \mathrm{K}}$ to equation (6).

The expression of success probability is obtained as

$$
\begin{aligned}
& \mathrm{p}_{\mathrm{s}}=\int_{0}^{\infty} 4 \pi \mathrm{r}_{1}^{2} \lambda_{0} \cdot \mathrm{e}^{-\frac{4}{3} \pi \mathrm{r}_{1}^{3} \lambda_{0}} \cdot \mathrm{e}^{-\frac{\gamma \sigma^{2} \mathrm{r}_{1}^{\alpha+1}}{\mathrm{P}_{\mathrm{t}} \mathrm{K}}} \\
& \cdot \mathrm{e}^{-4 \pi \lambda_{0} \int_{\mathrm{r}_{1}}^{\infty} \frac{\gamma \mathrm{r}_{1}^{\alpha+1}}{\gamma \mathrm{r}_{1}^{\alpha+1}+\mathrm{r}_{\mathrm{i}}^{(\alpha+1)}} \mathrm{r}_{\mathrm{i}}^{2} \mathrm{dr}_{\mathrm{i}}} \mathrm{dr}_{1} .
\end{aligned}
$$

When $\alpha=5$, the success probability can be simplified as

$$
\begin{aligned}
\mathrm{p}_{\mathrm{s}}=\int_{0}^{\infty} 4 \pi \mathrm{r}^{2} \lambda_{0} \cdot \mathrm{e}^{-\frac{4}{3} \pi \mathrm{r}^{3} \lambda_{0}} \cdot \mathrm{e}^{-\frac{\gamma \sigma^{2} \mathrm{r}_{1}^{6}}{\mathrm{P}_{\mathrm{t}} \mathrm{K}}} \\
\cdot \mathrm{e}^{-\frac{4}{3} \pi \lambda_{0 \sqrt{\mathrm{T}}} \mathrm{r}_{1}^{3}\left(\frac{\pi}{2}-\arctan \frac{1}{\sqrt{\mathrm{T}}}\right)} \mathrm{dr}_{1} .
\end{aligned}
$$

Proof: When $\alpha=5, p_{s}$ can be expressed as

$$
\begin{aligned}
& \mathrm{p}_{\mathrm{s}}=\int_{0}^{\infty} 4 \pi \mathrm{r}^{2} \lambda_{0} \cdot \mathrm{e}^{-\frac{4}{3} \pi \mathrm{r}_{1}^{3} \lambda_{0}} \cdot \mathrm{e}^{-\frac{\gamma \sigma^{2} \mathrm{r}_{1}^{6}}{\mathrm{P}_{\mathrm{t}} \mathrm{K}}} \\
& \cdot \mathrm{e}^{-4 \pi \lambda_{0} \int_{\mathrm{r}_{1}}^{\infty} \frac{\gamma \mathrm{r}_{1}^{6}}{\gamma \mathrm{r}_{1}^{6}+\mathrm{r}_{\mathrm{i}}^{6}} \mathrm{r}_{\mathrm{i}}^{2} d \mathrm{r}_{\mathrm{i}}} \mathrm{dr} \mathrm{r}_{1} \\
& \underline{(a)} \int_{0}^{\infty} 4 \pi r^{2} \lambda_{0} \cdot \mathrm{e}^{-\frac{4}{3} \pi \mathrm{r}_{1}^{3} \lambda_{0}} \cdot \mathrm{e}^{-\frac{\gamma \sigma^{2} \mathrm{r}_{1}^{6}}{\mathrm{P}_{\mathrm{t}} \mathrm{K}}} \\
& \cdot \mathrm{e}^{-\frac{4}{3} \pi \lambda_{0} \int_{\mathrm{r}_{1}^{3}}^{\infty} \frac{\gamma \mathrm{r}_{1}^{6}}{\gamma \mathrm{r}_{1}^{6}+\mathrm{x}^{2}} \mathrm{dx}} \mathrm{dr} \mathrm{r}_{1} \\
& =\int_{0}^{\infty} 4 \pi r^{2} \lambda_{0} \cdot e^{-\frac{4}{3} \pi r^{3} \lambda_{0}} \cdot e^{-\frac{\gamma \sigma^{2} r_{1}^{6}}{\mathrm{P}_{\mathrm{t}} \mathrm{K}}} \\
& \text {. } e^{-\frac{4}{3} \pi \lambda \lambda_{0 \sqrt{T}} r_{1}^{3}\left(\frac{\pi}{2}-\arctan \frac{1}{\sqrt{T}}\right)}{d r_{1}}_{1} \text {, }
\end{aligned}
$$

where step (a) follows by using the change of variables $r_{i}^{3} \rightarrow x$. When $\sigma^{2}=0$, the success probability is only related to the SINR threshold $\gamma$, and it is expressed as

$$
\mathrm{p}_{\mathrm{s}}=\int_{0}^{\infty} \mathrm{e}^{-\int_{\mathrm{y}}^{\infty} \frac{\gamma \mathrm{y}^{\frac{\alpha+1}{3}}}{\gamma \mathrm{y}^{\frac{\alpha+1}{3}}+\mathrm{x}^{\frac{\alpha+1}{3}}} \mathrm{dx}} \cdot \mathrm{e}^{-\mathrm{y}} \mathrm{dy} .
$$

Proof: When $\sigma^{2}=0, p_{s}$ can be expressed as

$$
\begin{aligned}
& \mathrm{p}_{\mathrm{s}}=\int_{0}^{\infty} 4 \pi \mathrm{r}_{1}^{2} \lambda_{0} \cdot \mathrm{e}^{-4 \pi \lambda_{0} \int_{\mathrm{r}_{1}}^{\infty} \frac{\gamma \mathrm{r}_{1}^{\alpha+1}}{\gamma \mathrm{r}_{1}^{\alpha+1}+\mathrm{r}_{\mathrm{i}}^{(\alpha+1)}} \mathrm{r}_{\mathrm{i}}^{2} \mathrm{dr}_{\mathrm{i}}} \\
& \text { - } \mathrm{e}^{-\frac{4}{3} \pi \mathrm{r}_{1}^{3} \lambda_{0}} \mathrm{dr}_{1} \\
& =\int_{0}^{\infty} e^{-\int_{r_{1}}^{\infty} \frac{\gamma r_{1}^{\alpha+1}}{\gamma r_{1}^{\alpha+1}+r_{i}^{(\alpha+1)}} d \frac{4}{3} \pi r_{i}^{3} \lambda_{0}} \\
& \cdot e^{-\frac{4}{3} \pi r_{1}^{3} \lambda_{0}} d \frac{4 \pi r_{1}^{3} \lambda_{0}}{3_{\alpha+1}} \\
& \underline{\underline{(a)}} \int_{0}^{\infty} \mathrm{e}^{-\int_{\mathrm{y}}^{\infty} \frac{\gamma \mathrm{y}^{\frac{\alpha+1}{3}}}{\gamma \mathrm{y}^{\frac{\alpha+1}{3}}+\mathrm{x}^{\frac{\alpha+1}{3}}} \mathrm{dx}} \cdot \mathrm{e}^{-\mathrm{y}} \mathrm{dy},
\end{aligned}
$$

step (a) is obtained by using the change of variables $\frac{4 \pi r_{1}^{3} \lambda_{0}}{3} \rightarrow x$ and $\frac{4}{3} \pi r_{i}^{3} \lambda_{0} \rightarrow y$.

The no noise $\left(\sigma^{2}=0\right)$ result is the upper bound of success probability since the noise-related part in success probability is $e^{-\frac{\gamma \sigma^{2} r_{1}^{\alpha+1}}{P_{t} K}}$, and $e^{-\frac{\gamma \sigma^{2} r_{1}^{\alpha+1}}{P_{t} K}} \leq\left. e^{-\frac{\gamma \sigma^{2} r_{1}^{\alpha+1}}{P_{t} K}}\right|_{\sigma^{2}=0}=1$.

As discussed at the beginning of this section, the number of effective serving BSs whose signals arrive with sufficiently - high SINRs also strongly affects the accuracy of the localization. The expression of success probability, i.e. the probability for a typical serving BS to successfully participate in the localization procedure is derived, and based on the system model, there is $n_{s f}$ serving BSs, therefore, the average number of effective serving BSs which can successfully participate in the localization procedure can be calculated as follows

$$
\mathrm{E}\left[\mathrm{N}_{\mathrm{bs}}\right]=\mathrm{p}_{\mathrm{s}} \cdot \mathrm{n}_{\mathrm{sf}} \text {. }
$$

\section{SIMULATION RESULTS}

Here, both numerical and Monte Carlo simulations are carried out to validate conclusions, and we use the default values in Table I obtained from [3] unless otherwise stated.

Table 1. Parameters of simulation

\begin{tabular}{|c|c|}
\hline Parameter & Value \\
\hline Path loss exponent & $\alpha=5$ \\
\hline Transmit power & $P_{t}=10 \mathrm{~W}$ \\
\hline $\begin{array}{c}\text { Constant attenuation per unit } \\
\text { of path length }\end{array}$ & $\mu=0.5$ \\
\hline Noise power & $\sigma^{2}=-70 \mathrm{dBm}$ \\
\hline
\end{tabular}

Fig. 3 compares the performance of average interference with different subframe numbers $n_{s f}$. It is shown that the average interference is an increasing function with BS density, which is because as the BS density increases, the number of BSs that may cause interference increases. In addition, when the number of subframe $n_{s f}$ increases, the average interference decreases, the

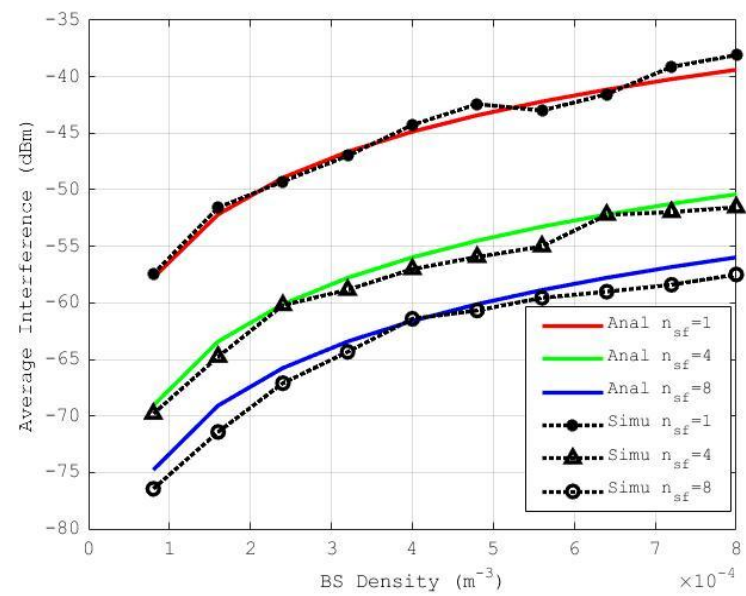

Figure 3. Average interference of different number of subframes vs. BS density.

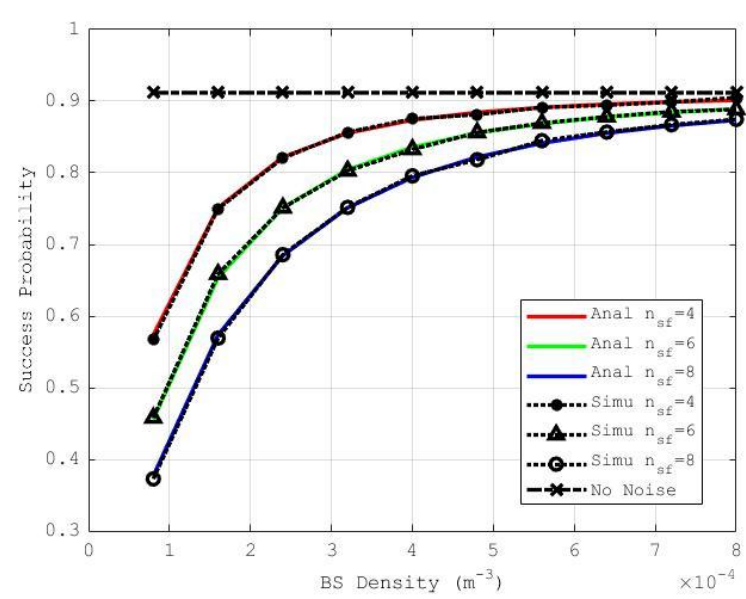

Figure 4. Success probability of different number of subframes vs. BS density. $\gamma=-10 \mathrm{~dB}$ 
reason is that when the BS density is the same, the increase in $n_{s f}$ can reduce the number of BS transmitting at the same time. Particularly, $n_{s f}=1$ can be regarded as the traditional no-time hopping condition. Therefore, it proves the conclusion that time hopping can effectively reduce the interference in TDOA. The analytical results match well with the corresponding simulation results.

Fig. 4 and Fig. 5 show the success probability performance with different value of $n_{s f}$. All the analytical results are validated with the simulation results.

Fig. 4 shows the success probability performances versus BS densities. It is shown that the success probability is an increasing function with BS density, which is because the useful signal will be stronger as the BS density increases. As the BS density keeps increasing, there will be a region, where a great increment in the BS density $\lambda$ leads to a negligible growth of the success probability, which is because when the BS density is large enough, not only the useful signal becomes stronger, the interference will also getting significantly stronger. It's illustrates in this figure that the success probability converges to the fixed value of $p_{s}$ without

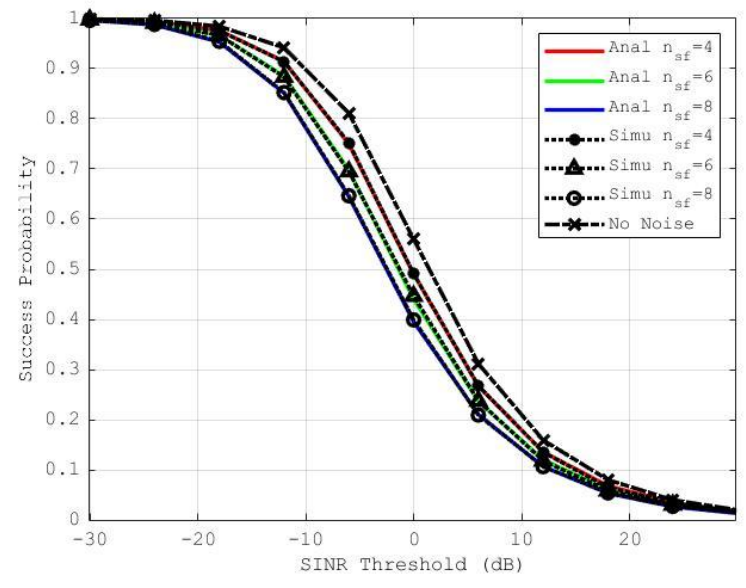

Figure 5. Success probability of different number of subframes vs. SINR threshold. $\lambda=0: 0004 \mathrm{~m}^{-3}$

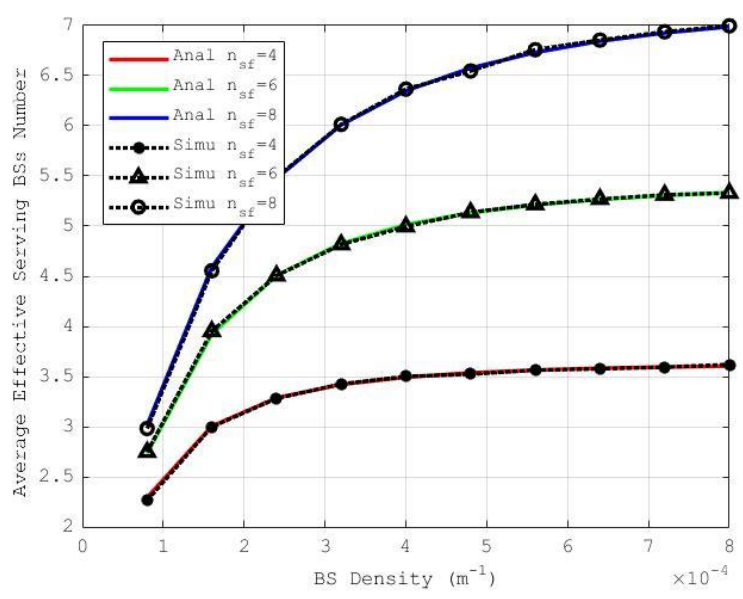

Figure 6. Average number of effective serving BSs of different number of subframes vs. BS density. $\gamma=-10$ dBFigure 1. A topology of 3D Poisson distributed base any noise as we have proved. The reason is as BS density continued to increase, the interference will become the main influence to the success probability and the noise can be neglected. In addition, the success probability is not improving with the increase of $n_{s f}$. The reason is when $n_{s f}$ increases the BSs density in a collection will decrease, in this case, although the interference is reduced, the useful signal is also reduced.

Fig. 5 shows the relationship between success probability and SINR threshold. As shown in this figure, the success probability decreases monotonically with respect to SINR threshold $\gamma$. The reason is that when $\gamma$ increases, it is more difficult to meet the SINR restriction for successfully participating in the localization process. In addition, the no noise case is the upper bound of the success probability as we have proved.

In Fig. 6 and Fig. 7, the average number of effective serving BS with sufficiently-high SINR is shown, and all the analytical results matches well with the Monte Carlo simulation results.

Fig. 6 shows the average number of effective serving BS versus BS density. The average number of effective serving BS is an increasing function with respect to BS density, which is because when the BS density increases, the success probability increases as shown in Fig. 4, and when the number of standby serving BS (the closest BSs in each collection, i.e. the BSs are selected as the serving BSs) is the same, the higher the success probability is, the greater the number of effective serving BS will be. It illustrates in this figure that the increase of $n_{s f}$ can improve the average number of effective serving BS. The reason is as $n_{s f}$ increases, the number of standby serving BS will increase.

In Fig. 7, it is shown the average number of effective serving BS is a decreasing function with respect to SINR threshold, which is because when the SINR threshold increases, less standby serving BS can meet the SINR restriction.

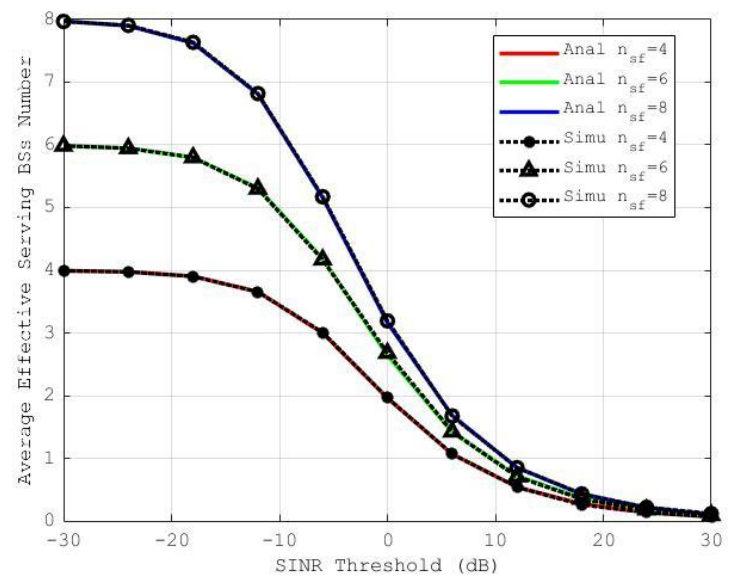

Figure 7. Average number of serving BSs of different number of subframes vs. SINR threshold. $\lambda=0: 0004 \mathrm{~m}^{-3}$

\section{CONCLUSION}

In this paper, an analytical model of time hopping based TDOA technology is proposed, and the performance of the proposed technology in $3 \mathrm{D}$ wireless networks is mathematically formulated. Firstly, the expression of average interference is derived. Based on that, the success probability and the number of effective 
serving BS are modeled and analyzed. In addition, some special cases are researched, which reveals interesting insights. Numerical results demonstrate that the time hopping can effectively reduce the interference, and a greater $n_{s f}$ can bring more benefits. All the results in this paper are validated with the Monte Carlo simulation results.

\section{ACKNOWLEDGMENTS}

This research was funded by the Science and Technology Planning Project of Guangdong Province of China (No. 2017B090908005), and the National Key R\&D Plan of China (No. 2016YFB0502001 and No. 2016YFB0502003).

\section{REFERENCES}

[1] M. Nozawa, Y. Hagiwara, and Y. Choi. 2012. Indoor human navigation system on smartphones using viewbased navigation. In 2012 12th International Conference on Control, Automation and Systems. 1916-1919.

[2] F. Jiang, Y. Kuang and 3. Åström, 2013. Time delay estimation for TDOA self-calibration using truncated nuclear norm regularization. In 2013 IEEE International Conference on Acoustics, Speech and Signal Processing. 3885-3889. DOI=http://dx.doi.org/10.1109/ICASSP.2013.6638386.

[3] Z. Deng, X. Yuan, Y. Yu, and X. Liu. 2012. Study of antiMAI self correlation peak detection based on terrestrial broadcasting systems. In 2012 IEEE 11th International Conference on Signal Processing, Vol. 1. 188-192. DOI=http://dx.doi.org/10.1109/ICoSP.2012.6491632.

[4] E. Staudinger and C. Gentner. 2011. TDoA subsample delay estimator with multiple access interference mitigation and carrier frequency offset compensation for OFDM based systems. In 2011 8th Workshop on Positioning, Navigation and Communication. 33-38. DOI=http://dx.doi.org/10.1109/WPNC.2011.5961011.

[5] L. Ruschendorf. 2010. D. Stoyan W. Kendall J. Mecke. Stochastic geometry and its applications. Crystal Research \& Technology 31, 6 (2010), 762-762.
[6] J. G. Andrews, F. Baccelli, and R. K. Ganti. 2011. A Tractable Approach to Coverage and Rate in Cellular Networks. IEEE Transactions on Communications 59, 11 (November 2011), 3122-3134. DOI=http://dx.doi.org/10.1109/TCOMM.2011. 100411.100541.

[7] Z. Pan and Q. Zhu. 2015. Modeling and Analysis of Coverage in 3-D Cellular Networks. IEEE Communications Letters 19, 5 (May 2015), 831-834. DOI=http://dx.doi.org/10.1109/LCOMM.2015.2411599.

[8] J. Schloemann, H. S. Dhillon, and R. M. Buehrer. 2016. Toward a Tractable Analysis of Localization Fundamentals in Cellular Networks. IEEE Transactions on Wireless Communications 15, 3 (March 2016), 1768-1782. DOI=http://dx.doi.org/10.1109/TWC.2015.2496273.

[9] Martin Haenggi. 2012. Stochastic Geometry for Wireless Networks — Interference and outage in wireless networks. 10.1017/CBO9781139043816, 5 (2012), 93-118.

[10] S. Y. Seidel and T. S. Rappaport. 1992. 914 MHz path loss prediction models for indoor wireless communications in multifloored buildings. IEEE Transactions on Antennas and Propagation 40, 2 (Feb 1992), 207-217. DOI=http://dx.doi.org/10. 1109/8.127405.

[11] Yi Tian Chen and Y. U. Ai min. 2005. Analysis of $2.4 \mathrm{GHz}$ WLAN Indoor and Outdoor Transmission Path Loss. Telecommunication Engineering (2005).

[12] H. ElSawy, E. Hossain, and M. Haenggi. 2013. Stochastic Geometry for Modeling, Analysis, and Design of MultiTier and Cognitive Cellular Wireless Networks: A Survey. IEEE Communications Surveys Tutorials 15, 3 (third 2013), 996-1019.

DOI=http://dx.doi.org/10.1109/SURV.2013.052213.00000. 